\title{
Apelin modulates pathological remodeling of lymphatic endothelium after myocardial infarction
}

\author{
Florence Tatin, ${ }^{1}$ Edith Renaud-Gabardos, ${ }^{1}$ Anne-Claire Godet, ${ }^{1}$ Fransky Hantelys, ${ }^{1}$ \\ Francoise Pujol, ${ }^{1}$ Florent Morfoisse, ${ }^{1}$ Denis Calise, ${ }^{2}$ Fanny Viars, ${ }^{3}$ Philippe Valet, ${ }^{1}$ Bernard Masri, ${ }^{1}$ \\ Anne-Catherine Prats, ${ }^{1}$ and Barbara Garmy-Susini ${ }^{1}$ \\ 'I2MC INSERM UMR 1048, Toulouse Cedex, France. UMS 006, INSERM, UPS, F-31432 Toulouse, France. \\ ${ }^{3}$ MetaToul-Lipidomique Core Facility, I2MC INSERM 1048, Toulouse, France.
}

\begin{abstract}
Lymphatic endothelium serves as a barrier to control fluid balance and immune cell trafficking to maintain tissue homeostasis. Long-term alteration of lymphatic vasculature promotes edema and fibrosis, which is an aggravating factor in the onset of cardiovascular diseases such as myocardial infarction. Apelin is a bioactive peptide that plays a central role in angiogenesis and cardiac contractility. Despite an established role of apelin in lymphangiogenesis, little is known about its function in the cardiac lymphatic endothelium. Here, we show that apelin and its receptor APJ were exclusively expressed on newly formed lymphatic vasculature in a pathological model of myocardial infarction. Using an apelin-knockout mouse model, we identified morphological and functional defects in lymphatic vasculature associated with a proinflammatory status. Surprisingly, apelin deficiency increased the expression of lymphangiogenic growth factors VECF-C and VEGF-D and exacerbated lymphangiogenesis after myocardial infarction. Conversely, the overexpression of apelin in ischemic heart was sufficient to restore a functional lymphatic vasculature and to reduce matrix remodeling and inflammation. In vitro, the expression of apelin prevented the alteration of cellular junctions in lymphatic endothelial cells induced by hypoxia. In addition, we demonstrated that apelin controls the secretion of the lipid mediator sphingosine-1-phosphate in lymphatic endothelial cells by regulating the level of expression of sphingosine kinase 2 and the transporter SPNS2. Taken together, our results show that apelin plays a key role in lymphatic vessel maturation and stability in pathological settings. Thus, apelin may represent a novel candidate to prevent pathological lymphatic remodeling in diseases.
\end{abstract}

Conflict of interest: The authors have declared that no conflict of interest exists.

Submitted: March 8, 2017

Accepted: May 10, 2017

Published: June 15, 2017

Reference information: JCI Insight. 2017;2(12):e93887. https://doi.org/10.1172/jci. insight. 93887

\section{Introduction}

Preserving the vascular lymphatic barrier is essential to maintain tissue fluid homeostasis and immune cell trafficking to perform immune surveillance. The integrity of the lymphatic vasculature relies on the adhesive and mechanical properties of specialized junctions on lymphatic capillaries that allow unidirectional entry of fluids, solutes, and immune cells draining into larger collecting vessels. A defect in the lymphatic drainage is associated with severe edema, which is an aggravating factor in a large spectrum of pathologies such as heart ischemia, lymphedema, and chronic inflammatory diseases (1).

In particular, recent literature demonstrated that lymphatic drainage plays a major role in myocardial infarction (MI) (2). MI is caused by a left coronary obstruction that leads to an orchestrated series of events, initiated by immune cell infiltration (inflammatory phase), and followed by edema and an adverse left ventricular remodeling, chronic inflammation, fibrosis, and angiogenesis. Ischemic heart exhibits a dysfunctional lymphatic network that participates in the development of chronic myocardial edema and aggravates cardiac dysfunction $(2,3)$. Therefore, improving the integrity of the lymphatic vascular wall remains an emerging challenge in cardiovascular biology. VEGF-A is the first vascular growth factor identified to improve angiogenesis after MI (4). However, phase II clinical trials have shown that VEGF-A is not efficient in improving the contractile function of ischemic heart (5). To date, our understanding about the molecular mechanisms leading to a restricted activation of lymphatic vessels in diseases has been mainly associated with VEGF-C/VEGFR-3 signaling. VEGF-C, the major lymphangiogenic growth factor 
that binds its receptor VEGFR-3, restores a functional lymphatic vasculature in models of lymphedema, chronic inflammatory bowel diseases, and lung allografts (6-8). Recently, VEGF-C appeared ineffective in improving lymphatic function in a mouse model of $\mathrm{MI}(2)$. Altogether, these observations demonstrate that identifying novel actors in the maintenance of vascular integrity remains a crucial challenge to restore heart function. In particular, the lipid mediator sphingosine-1-phosphate (S1P) has recently emerged as a potent mediator of vascular integrity $(9,10)$. In addition, the bioactive peptide apelin is a critical player in both lymphangiogenesis and angiogenesis (11-13). Apelin promotes lymphatic development in zebrafish (14) and, despite normal embryonic lymphatic vessel development in apelin-deficient mice (15), a link between apelin and the lymphatic endothelium has been clearly established in pathological models. Apelin promotes tumor lymphangiogenesis and lymph node metastasis (16). In diet-induced obesity mouse models, the lack of apelin leads to hyperplasia and abnormal leakiness of lymphatic vasculature, suggesting a role in maintaining lymphatic wall integrity (17).

In the present study, we demonstrate that apelin plays a key role in cardiac lymphatic vasculature. Our data reveal that apelin-deficient mice exhibit abnormal dilated and leaky lymphatic vasculature associated with a proinflammatory status. Conversely, we provide evidence that in the pathological setting of MI, overexpression of apelin protects the lymphatic vasculature organization and function and its cardiac microenvironment. In addition, we found that apelin regulates the secretion of S1P in lymphatic endothelial cells (LECs) and maintains the integrity of cellular junctions. Therefore, our work highlights potentially novel perspectives to counteract pathological remodeling of lymphatic vasculature in a broad array of inflammatory and cardiovascular diseases.

\section{Results}

The cardiac lymphatic system. To obtain a comprehensive view of the lymphatic drainage in adult heart, we first performed paraffin and cryosection analyses along the heart from the apex through the atrioventricular (AV) junction. Most of the lymphatic vessels were located at the epicardial and subepicardial surface of the heart (Figure 1, A and B, and Supplemental Figure 1A; supplemental material available online with this article; https://doi.org/10.1172/jci.insight.93887DS1). A low-density lymphatic network starting at the apex progressively appeared at higher density to cover the entire epicardial surface along the ventricular region (Figure 1B and Supplemental Figure 1B). Larger lymphatic vessels were seen in the epicardium and in the vicinity of the right ventricle septum, whereas the myocardium contained mainly capillaries defined as LYVE-1 positive but CD45 negative (Figure 1C and Supplemental Figure 1C). Interestingly, we found the presence of lymphatic vessels in the tricuspid and mitral valves (Figure 1D), suggesting a potential role of the lymphatic vasculature in the pathophysiology of cardiac valvular diseases. To further define the identity of cardiac LECs, we performed the immunodetection of lymphatic vessels using podoplanin, VEGFR-3, and NRP2 antibodies. We observed that all of the LEC markers were found in larger lymphatic vessels, whereas podoplanin was absent from myocardial lymphatic capillaries (Figure 1E and Supplemental Figure 1D). Based on our findings, we could envision a bidirectional drainage of the heart: a centrifugal drainage from the myocardium to the subepicardium and an ascending drainage from the apex to the AV junction. This hypothesis was confirmed by the visualization of Evan's blue drainage injected in the apex or in the myocardium (Figure 1F). The lymph drainage is represented in the schematic cartoon in Figure 1G.

MI induces a long-term dysfunctional lymphatic network. To examine the post-MI lymphatic remodeling, we generated a mouse model of MI with reduced cardiac systolic function (Supplemental Table 1) and performed whole-mount staining of cardiac lymphatic vessels with an LYVE-1 antibody. We observed 2 weeks after MI a necrotic area in the left ventricle corresponding to the ischemic zone with a rarefaction of the lymphatic system, whereas the right ventricle lymphatic system was not affected (Figure 2A). Six weeks after MI, the left ventricular wall thickness was increased due to cardiac remodeling. The lymphangiogenic process appeared disorganized with abnormal vessel morphology in the infarcted zone. Surprisingly, the growth of dilated and tortuous lymphatic vessels extended throughout the right ventricle, indicating a strong stimulation of the lymphatic vasculature beyond the ischemic zone (Figure 2A). Moreover, we examined in greater detail the remodeling of lymphatic vasculature by 2-photon microscopy using LYVE-1 immunostaining associated with the second harmonic generation to observe the arrangement of collagen fibers (Figure 2B). Two weeks after MI, disrupted lymphatic vessels were observed close to the infarcted zone, while lymphatic vessels in the ventral part were unaffected and associated with distinct collagen fibers. At 6 weeks after MI, the lymphatic network was disrupted with large, dysfunctional, and hyperplastic lymphatic 

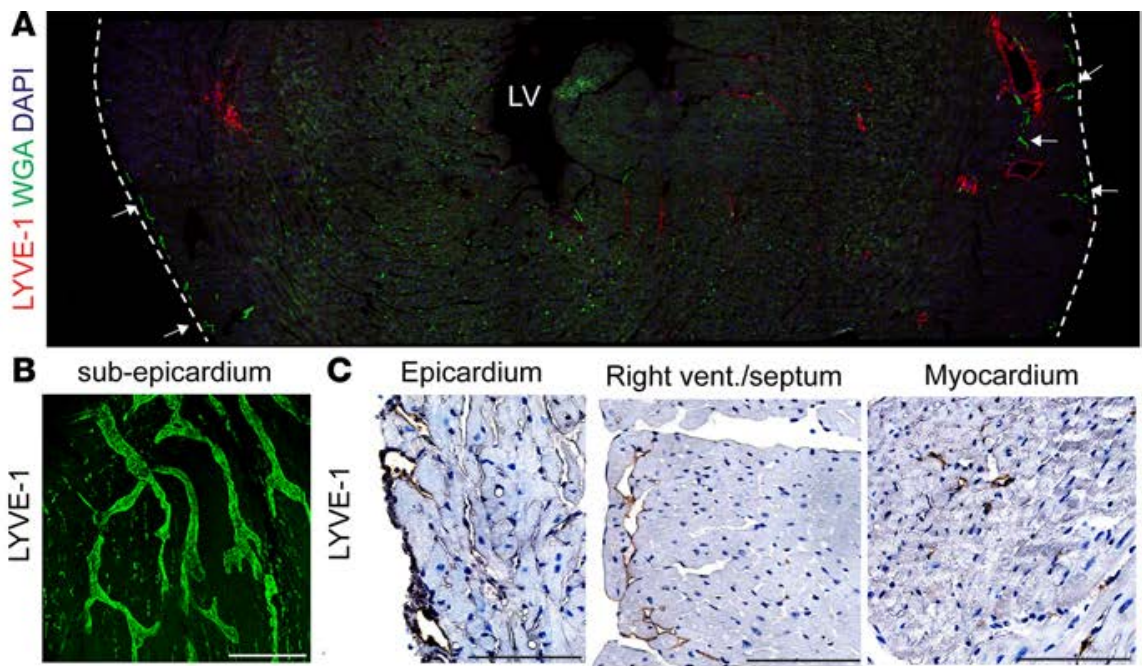

Myocardium

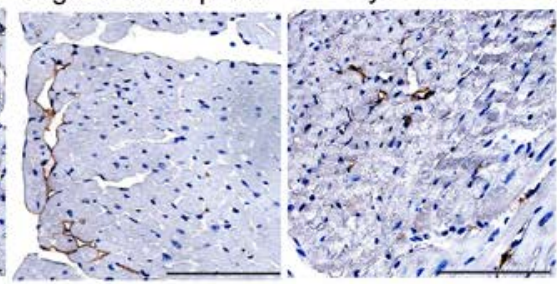

D

Right ventricle- Tricuspid valve
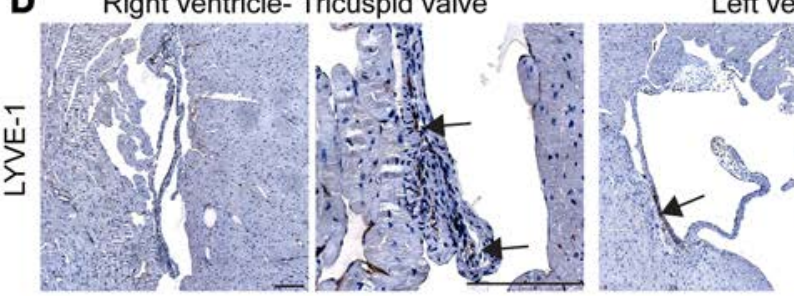

eft ventricle-mitral valve

$\mathbf{E}$

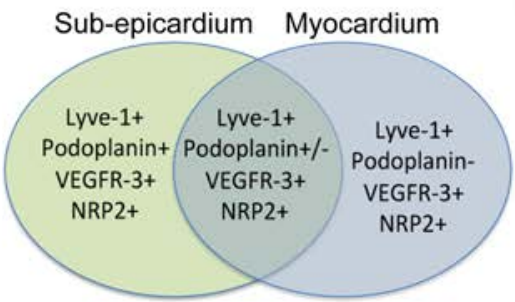

\section{$\mathbf{F}$}

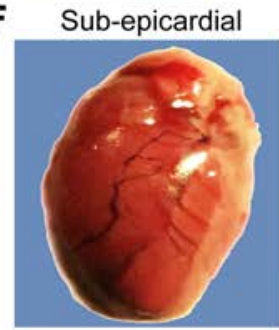

Figure 1. Characterization of lymphatic vasculature in adult heart. (A) Immunostaining of a transversal cross-section through the left ventricle (LV) to examine the localization of lymphatic vessels in heart with LYVE-1 antibody (green) and the lectin wheat germ agglutinin (WCA, red). Arrows show LYVE-1-positive lymphatic vessels. (B) Whole-mount imaging of LYVE-1-positive lymphatic vessels at the epicardial surface of the heart. (C and $\mathbf{D})$ Localization of capillaries and collecting lymphatic vessels with LYVE-1 antibody on paraffin cross-sections. Small lymphatic capillaries are observed in the myocardium, whereas larger vessels are located on the right ventricular side of the interventricular septum and on the subepicardial surface of the heart. Interestingly, we found the presence of lymphatic vessels at the base of mitral valve and in the tricuspid valve leaflets. (E) Identification of markers used to determine the cardiac lymphatic endothelium. NRP2, neuropilin 2; VEGFR3, vascular endothelial growth factor receptor 3 ; LYVE-1, lymphatic vessel endothelial hyaluronan receptor 1 . Capillaries in the myocardium are defined positive for all markers except podoplanin. Larger lymphatic vessels in the septum expressed podoplanin only in the superior part of the heart. (F) Drainage of lymphatic vasculature visualized by Evans blue dye. Evans blue was injected either at the subepicardial surface of the apex or intramyocardium. (G) Schematic representation of the heart lymphatic drainage. Scale bars: $250 \mu \mathrm{m}$ (B) and $100 \mu \mathrm{m}$ (C and D).

vessels associated with disorganized collagen fibers (Figure 2B). This information is in line with a dysfunctional drainage of lymphatic vessels previously reported in rat model of myocardial infarction (2). To further characterize the remodeling of post-MI lymphatic vessels, we performed immunodetection of the lymphatic vessels on heart cryosections after ischemia. We observed an increase of enlarged lymphatic vessels in the edge of the infarct zone 2 weeks after MI that developed through the right and left periventricular zones at 6 weeks after MI (Figure 2C and

Supplemental Figure 2A). We remarkably found that the developing lymphatic vasculature, positive for VEGFR3 and podoplanin, exhibited a dilated morphology in the infarct and noninfarct zone (Supplemental Figure 2, B-D and Figure 2C). We found that the cardiac lymphangiogenesis mainly occurred in the epicardium and rarely in the septum (Figure 2D). Importantly, this dilated morphology was maintained up to 6 weeks after MI (Figure 2, E and F), suggesting that long-term heart tissue injury generates the incapacity of lymphatic vessels to return to physiological morphology.

We next studied isolated cardiac LECs from mouse heart to define the molecular signature of lymphatic endothelium associated with cardiac remodeling. The entire heart, except for the atrium, was taken for analysis. We defined LECs in WT adult heart as CD $45^{-} \mathrm{CD} 31^{+}$Lyve- $1^{+}$by cytometric analysis. LECs represented $3.2 \%$ of the $\mathrm{CD} 45^{-} \mathrm{CD} 31^{+}$population, whereas $96.7 \%$ were blood endothelial cells (BECs) (Figure 2G). We confirmed the identity of cardiac LECs by the expression of the lymphatic markers Lyve-1, Prox-1, Pdpn (podoplanin), as well as Vegfr 3 by quantitative reverse transcription PCR (RT-qPCR) compared with BECs (Figure $2 \mathrm{H}$ ). Gene expression analysis was next performed in isolated LECs from ischemic 
A
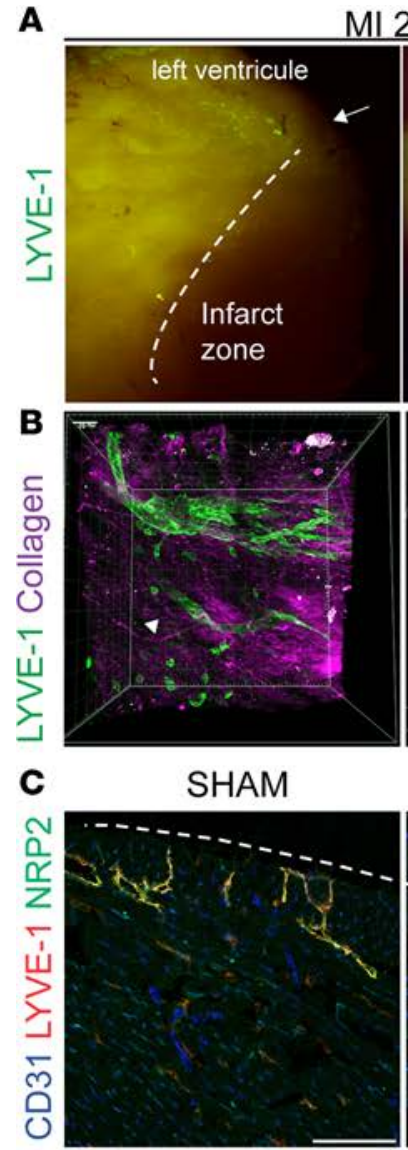

D

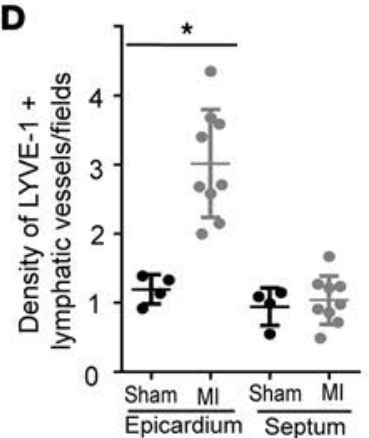

G

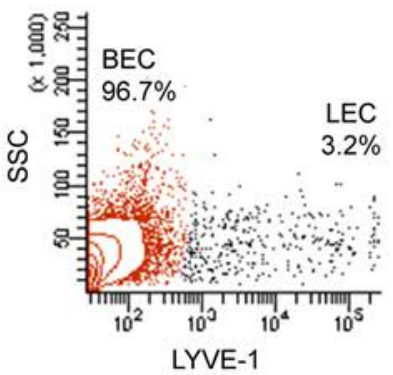

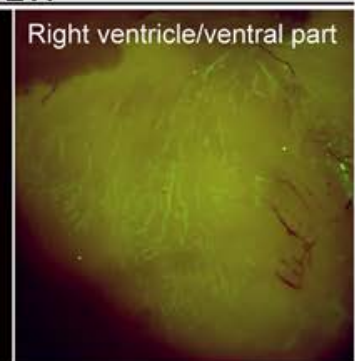

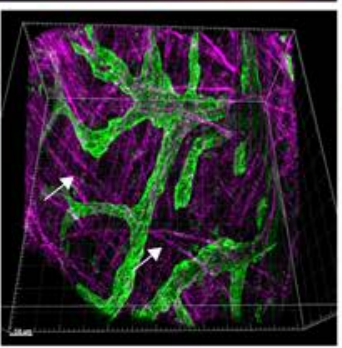

MI 2W
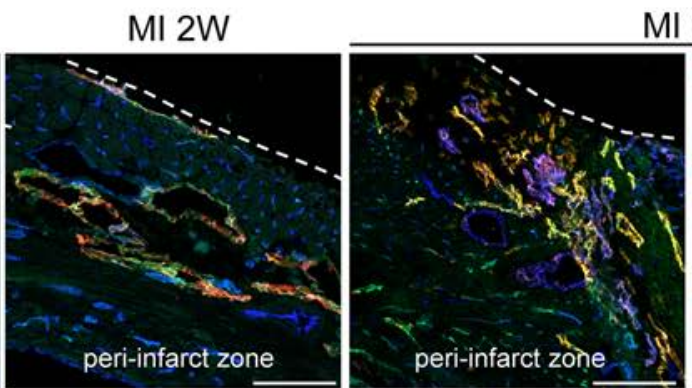

MI 6W
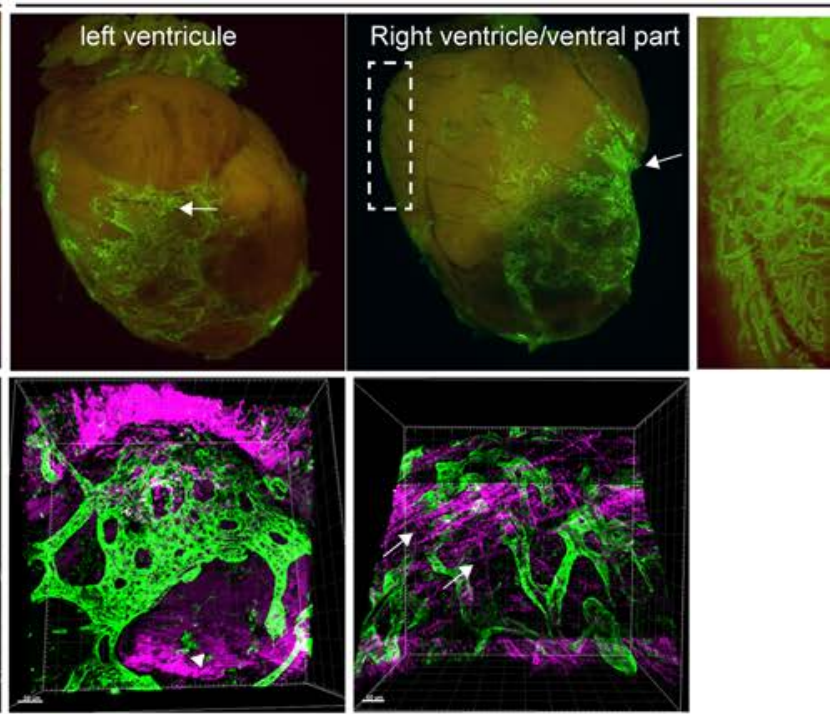

MI 6W

E

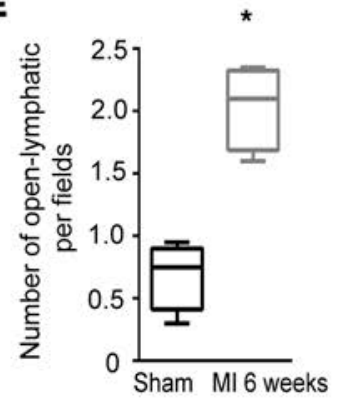

H

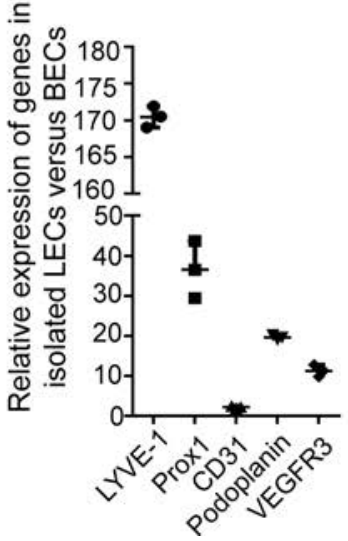

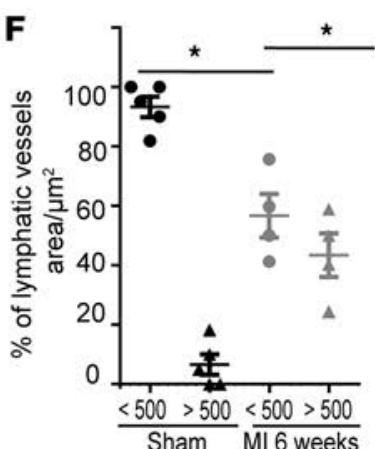

$\mathbf{I}$

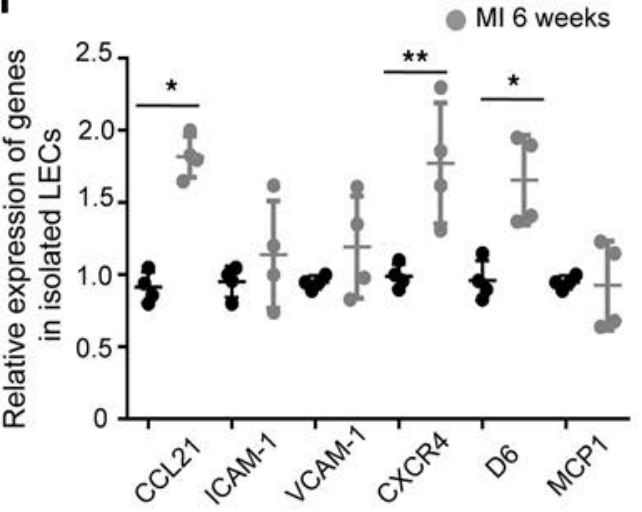

Figure 2. Chronic myocardial ischemia induces pathological remodeling of lymphatic vasculature. (A) Visualization of epicardial lymphatic vasculature by whole-mount heart immunostaining with LYVE-1 antibody 2 weeks or 6 weeks after myocardial infarction (MI). Dotted line indicates the limit of infarcted zone. Note the important remodeling of lymphatic vasculature that extends beyond the infarcted zone. Arrows indicate the position of the ligation. (B) Two-photon microscopic analysis of epicardial lymphatic vessels with LYVE-1 antibody. Two weeks after MI, the lymphatic network is disrupted 
and associated with disordered collagen fibers (arrowheads) close to the infarcted zone, while the lymphatic network on the ventral part is not affected. Note the presence of well-organized collagen fibers (arrows). Six weeks after MI, a strong, disorganized, and hyperplastic lymphatic network near the infarct zone that propagates to the opposite side of the MI can be seen. (C) Representative images of heart cross-section stained for LYVE-1, NRP2, and CD31 showing the remodeling of lymphatic vasculature after MI. Enlarged lymphatic vessels appear at the infarct border zone at 2 weeks after MI. Note that at 6 weeks after MI, dilated vessels are also present at a distance in the noninfarct zone. (D) LYVE-1-positive vessel density in the subepicardium and septum ( $n=4-9,20$ fields were counted per condition). ${ }^{*} P<0.004$. (E) Number of open lymphatic vessels located at the subepicardial surface of the heart in sham and 6 weeks after MI ( $n=4-9,20$ fields were counted per condition). ${ }^{*} P<0.0001$. (F) Area of lymphatic vessels expressed as percentage of vessels less than or greater than $500 \mu \mathrm{m}^{2}$ ( $n=4-5,40$ vessels were counted per condition). ${ }^{*} P<0.0017$. (G) Representative panel showing the endothelial cell (EC) populations defined as CD45- and CD31+. The LYVE-1 marker allows us to differentiate the lymphatic ECs (LECs) that represent only $3.2 \%$, while blood ECs (BECs) represent $96.7 \%$ in healthy heart. (H) Expression of known lymphatic markers in isolated cardiac LECs compared with BECs in normal heart. Nine or 10 hearts were pooled together to sort ECs; data represent mean \pm SD, $n=3$. (I) Gene expression in cardiac-isolated LECs from sham mice or 6 weeks after MI. Note the expression of prolymphangiogenic receptor CXCR4 and the expression of inflammatory mediators such as CCL21 and D6. Nine or 10 hearts were pooled together to sort ECs; data represent mean $\pm \mathrm{SD}, n=4$. ${ }^{*} P<0.001,{ }^{*} P<0.0005$. Statistical analysis was done with Student's $t$ test ( $\mathbf{D}$ and $\left.\mathbf{E}\right)$ or 1-way ANOVA with Bonferroni post-hoc test (F and I). Scale bars: $50 \mu \mathrm{m}$ (B and C).

heart compared with sham mouse heart. The lymphangiogenic receptor Cxcr4 was upregulated, indicating an activation of the lymphangiogenesis process at 6 weeks after MI. Interestingly, the inflammatory markers Icam-1, Vcam-1, and Mcp-1 were not upregulated; however, we noted the expression of the chemokine Ccl21 and the chemokine-scavenging receptor D6 (Figure 2I), suggesting an important role of lymphatic vasculature in modulating immune cell responses during cardiac remodeling.

Activated cardiac LECS express apelin and its receptor APJ. Despite a well-established role of apelin in the regulation of cardiac contractility and angiogenesis (18-20), its role in cardiac lymphatic endothelium has to the best of our knowledge never been explored. To address this issue, we first analyzed by RT-qPCR the expression of $A p \ln$ (apelin) as well as its receptor $A P J$ in the ischemic area 2 days, 1 week, and 1 month after MI (Figure 3, A and B). In parallel, we measured the expression of lymphangiogenic factors Vegf-C and Vegf-D and their receptor Vegfr3 (Figure 3C). We noted a striking expression of Apln 2 days after MI, whereas the long-term post-MI level of the receptor $A P J$ increased, as previously reported (21), suggesting an activation of the apelin/APJ signaling in early days through the reparative phase of MI. As expected, $V e g f-C$ and $V e g f-D$ expression was not induced 2 days after MI but progressively increased from 1 week to 4 weeks after MI, suggesting the stimulation of lymphangiogenesis at a later stage of MI (Figure 3C). The increase of Vegfr3 at 2 days after MI may correlate with its critical role in adaptive and innate immunity.

To investigate the role of apelin/APJ signaling in lymphatic endothelium, we next evaluated the localization of apelin and APJ expression in cardiac LECs by immunohistochemistry. Although neither protein was detected on quiescent cardiac lymphatic vessels (Figure 3D) or in early post-MI days (data not shown), we visualized the localization of the receptor APJ on activated lymphatic vessels 6 weeks after MI (Figure 3D). Similarly, apelin expression was partially associated with cardiac lymphatic vessels in post-MI heart (Figure 3D). Importantly, APJ was only observed in the growing lymphatic vessels within the infarct zone, suggesting a specific requirement of apelin/APJ signaling in a pathological microenvironment. We observed consistent heterogeneity in APJ expression on lymphatic endothelium where only a subset of cells forming lymphatic vessels expressed APJ. Therefore, we conclude that the receptor APJ is strongly regulated spatially and temporally in activated lymphatic endothelium. Taken together, our data document a role for apelin/APJ signaling in newly formed lymphatic vessels that undergo ischemic stress.

Abnormalities of lymphatic vasculature in apelin- $\mathrm{KO}$ mice are associated with a proinflammatory condition. To determine whether apelin regulates lymphatic vessel development, we initially analyzed the lymphatic vasculature in apelin-deficient mice. In agreement with the literature (15), the density of lymphatic vasculature at the subepicardial surface of the heart was similar in apelin-deficient mice and control mice. However, a prominent enlargement of lymphatic vasculature was observed in apelin-KO mice (Figure 4, A and B). Our finding was also confirmed in ear skin and back dermal skin tissue, indicating that the dilation of lymphatic vessels is an overall phenotype in apelin-KO mice and not organ specific (Supplemental Figure 3, A-D). The lack of apelin had no effect on the lymphatic vessel branching and density compared with control, whereas we noted an increase in the diameter of lymphatic vessels (Supplemental Figure 3, B and C). The presence of highly dilated lymphatic vessels in apelin-deficient mice suggests a disrupted lymphatic drainage leading to overloaded vessels (Supplemental Figure 3D). To evaluate whether changes in lymphatic morphology correlate with compromised lymphatic drainage, we performed skin lymphography by injecting FITC-dextran into mouse footpads. We observed prominent leakage of the lymphatic system associated with a dermal backflow of the dye in apelin-KO mice (Supplemental Figure 3E). 
A

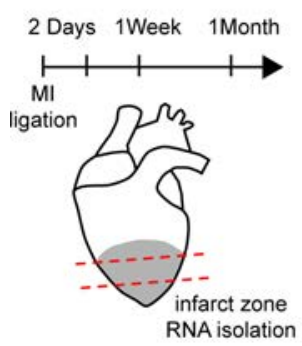

D

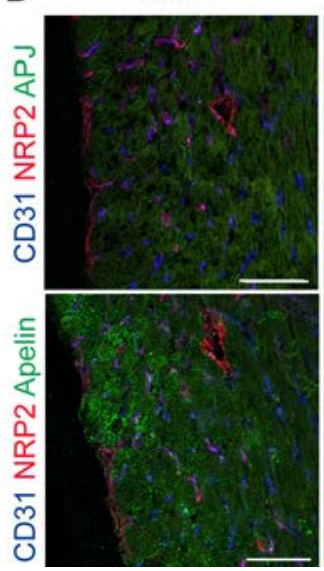

B
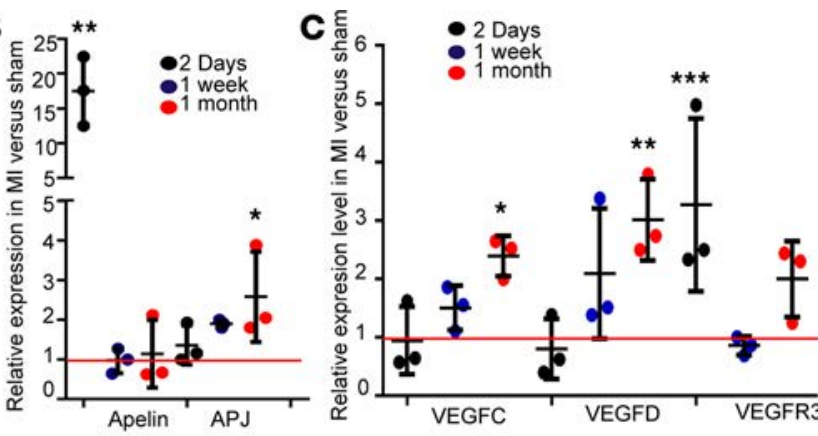

MI 6 weeks

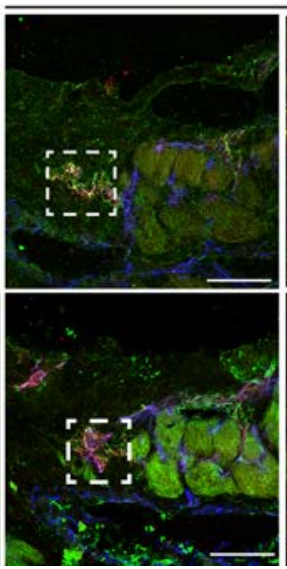

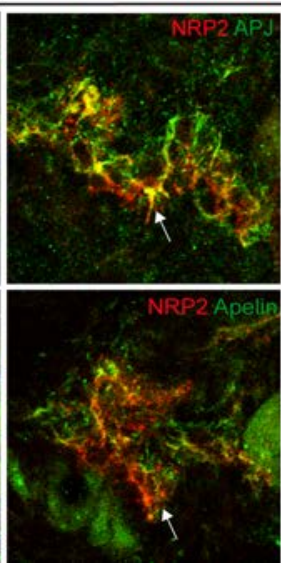

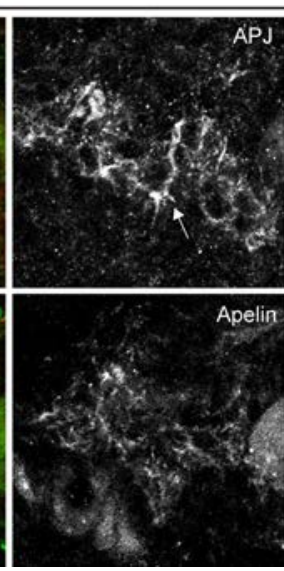

Figure 3. APJ is expressed on activated lymphatic endothelium in the ischemic zone. (A) Schematic representative of RNA isolation at the infarcted zone at different time points. (B) Relative expression of Apln (apelin) and the receptor $A P J$ after myocardial infarction (MI) analyzed by RT-qPCR. Data represent mean \pm SD, $n=3 .{ }^{*} P<0.05,{ }^{* *} P<0.0001$. (C) Relative expression of lymphangiogenic factors Vegf- $C$ and Vegf- $D$ and the receptor Vegfr-3 after MI. Data represent mean \pm SD. ${ }^{*} P<0.05,{ }^{* *} P<$ $0.003,{ }^{* * *} P<0.001$. (D) Localization of apelin and its receptor APJ on lymphatic endothelium in sham mice or 6 weeks after MI. Note the partial colocalization between apelin-positive and NRP2-positive lymphatic vessels, suggesting a paracrine action in lymphatic endothelium. Statistical analysis was done with 1-way ANOVA with Bonferroni post-hoc multiple comparison test. Scale bars: $50 \mu \mathrm{m}$ (D).

The lymphatic vasculature provides local information by facilitating the trafficking of immune cells from tissues to the lymph nodes. Hyperpermeability and enlarged lymphatic vessels suggest the development of basal tissue inflammation in apelin-deficient mice. Therefore, we analyzed the lymphatic vasculature in apelin-KO mice lymph nodes. Interestingly, apelin-KO mice have an expanded lymphatic network within inguinal and mesenteric lymph nodes, thus indicating the presence of a proinflammatory status (Supplemental Figure 4). We next used flow cytometric analysis to assess the distribution of immune cell populations in the heart of apelin-KO mice compared with control mice. Although we did not observe any change in the ratio of CD3-positive T lymphocytes, we found a consistent increase in the amount of CD19. positive B lymphocytes in apelin-KO $(27.3 \% \pm 3.4 \%)$ compared with control mice $(15.9 \% \pm 1.4 \%)$ (Figure $4, \mathrm{C}$ and $\mathrm{D})$. In addition, the ratio of $\mathrm{CD} 45^{+} \mathrm{CD} 11 \mathrm{~b}^{+} \mathrm{CD} 206^{+}$macrophages was reduced in correlation with an increase of the $\mathrm{CD} 45^{+} \mathrm{CD} 11 \mathrm{~b}^{+} \mathrm{CD} 206^{-} \mathrm{M} 1$-like macrophage subset in apelin-KO mice (Figure 4, $\mathrm{E}$ and $\mathrm{F}$ ). Our data indicate a change in the macrophage population towards an M1-like proinflammatory phenotype in apelin-KO mice. Interestingly, the macrophage population positive for CD45, CD11b, and LYVE-1 decreased in apelin-KO mice to $33.9 \% \pm 3.2 \%$ compared with $46.7 \% \pm 3.8 \%$ for control (Figure 4 , $\mathrm{E}$ and F). LYVE-1 ${ }^{+}$macrophages often located close to the lymphatic vasculature have been shown to play an important role in the morphogenesis and remodeling of the lymphatic vasculature (22). Taken together, these data reveal a previously undescribed remodeling of lymphatic vasculature in adult apelin-KO mice and support the idea of an important role of apelin in the maintenance of antiinflammatory status. Conversely, an imbalance of apelin level may favor the development of a proinflammatory condition associated with an enlarged and dysfunctional lymphatic network.

The lack of apelin exacerbates a proinflammatory response and worsens abnormalities of lymphatic vasculature in MI. Infiltration of immune cells and edema are hallmarks of early-stage MI. Therefore, we investigated the phenotype of lymphatic vasculature by immunostaining using LYVE-1 and CD31 antibodies 4 days after MI. Remarkably, we observed the presence of enlarged and hyperplastic lymphatic vessels in the subepicardium close to the peri-infarct or within the infarct zone (Figure 5A). In contrast, lymphatic density was not modified, suggesting a sudden adaptation of lymphatic vessels to edema in response to heart injury (data not shown). We have previously demonstrated that apelin is crucial in the maintenance of a functional lymphatic vasculature. We found that cardiac lymphatic vessels in apelin-deficient mice 4 days after MI 

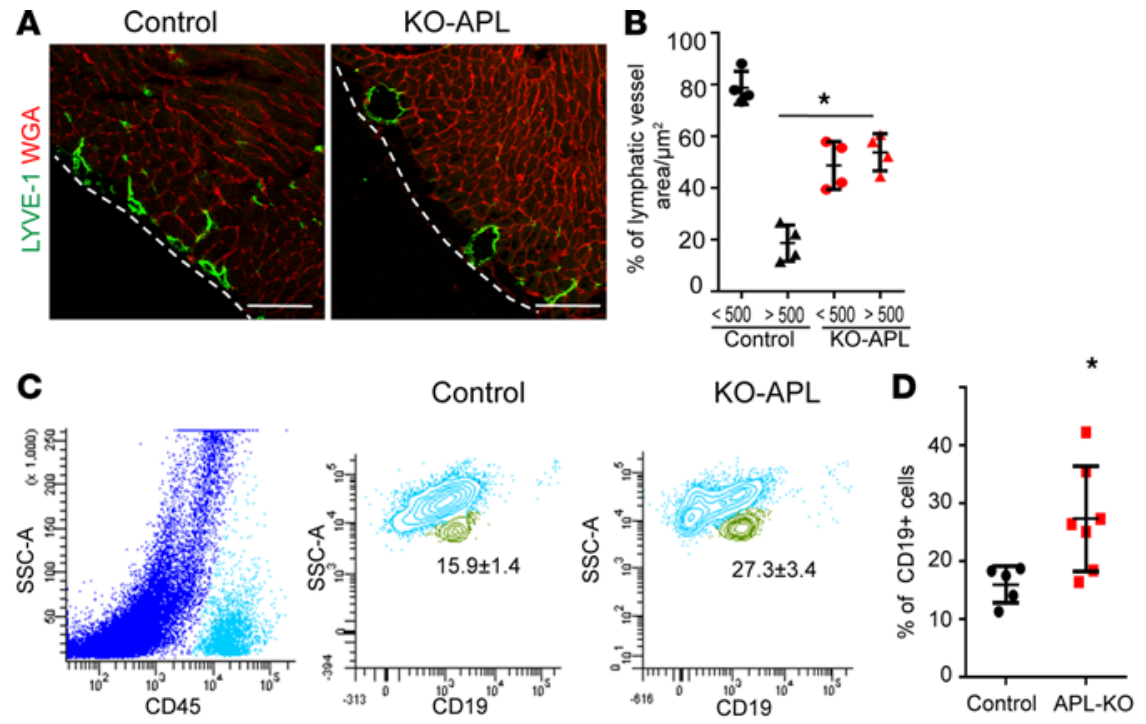

E

Control
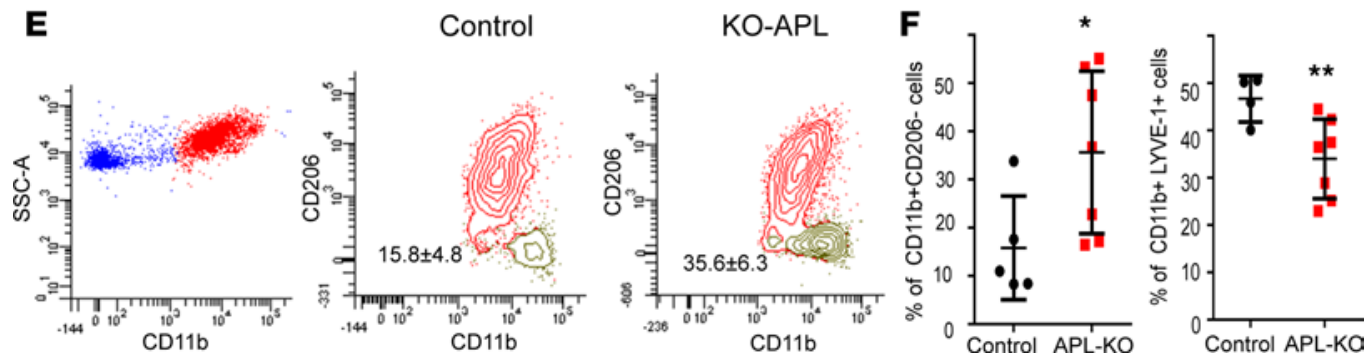

Figure 4. Apelin-KO mice develop abnormal lymphatic vasculature and a proinflammatory status. (A) Visualization of cardiac lymphatic vasculature (LYVE-1, green) and cardiomyocytes (wheat germ agglutinin [WGA], red) in apelin-KO versus control mice. Dilated lymphatic vessels were found in the subepicardial surface of the heart of adult apelin-KO mice. (B) Quantification of the area of lymphatic vessels in control and apelin-KO mice. The area of all lymphatic vessels located at the subepicardial surface was measured ( $n=4$ per group; values are percentage \pm SD). ${ }^{*} P<0.004$. (C) Representative panels of flow cytometric analysis of heart tissue showing the CD45+CD19+ population in apelin-KO mice compared with control. (D) Percentage of $\mathrm{CD}^{+} 9^{+}$in control and apelin-deficient mice. Note the increase in $\mathrm{CD}_{19}{ }^{+} \mathrm{B}$ lymphocytes in apelin-KO mice $(n=6-8$ per group, percentage CD45-positive cells $\pm \mathrm{SEM}$ ). ${ }^{*} P<0.02$. (E) Representative flow cytometric analysis of heart showing the increase in CD11b+CD206- in apelin-KO mice versus control. (F) Percentage of CD11b+CD206 ${ }^{-}$and CD11b+LYVE-1+ $(n=6-8$ per group, percentage of CD11b-positive cells $\pm \mathrm{SEM}) .{ }^{*} P<0.04,{ }^{* *} P<0.02$. Statistical analysis was done with Student's $t$ test. Scale bars: $100 \mu \mathrm{m}(\mathbf{A})$. APL, apelin.

exhibited dilated morphology as observed in control mice, which is probably due to the maximum capacity of lymphatic vessels reached in apelin-KO mice (Supplemental Figure 5). Remarkably, the infiltration of $\mathrm{CD} 68^{+}$macrophages and the expression of the proinflammatory cytokine TNF- $\alpha$ were greatly increased in apelin-KO mice after acute MI (Figure 5, B and C). In agreement with these results, we found that apelindeficient mice displayed a 6-fold increase in CCL21 expression and a 2-fold increase in the expression of the lymphangiogenic factors VEGF-C and VEGF-D compared with control mice (Figure 5D). These results correlated with an increase in lymphatic vessel density 6 weeks after MI (Figure 5E). Taken together, our data indicate that the lack of apelin potentiates a proinflammatory response in the acute phase of MI.

Apelin regulates lymphatic vessel maturation and cardiac homeostasis. We next aimed to clarify a potential beneficial role of apelin after MI and generated an apelin-overexpressing lentivector to transduce the myocardium. In our model, long-term overexpression of apelin was not sufficient to significantly improve cardiac function, yet we measured a consistent decrease in cardiac fibrosis (Figure 6, A and B, and Supplemental Figure 6, A and B). To further decipher the molecular mechanisms underlying apelin's role in cardiac ischemia, we performed gene expression analysis related to cardiac hypertrophy, matrix remodeling, and inflammation. Atrial natriuretic peptide (ANP) and brain natriuretic peptide (BNP) expression are upregulated in conditions of heart failure, cardiac hypertrophy, and fibrosis (23). We found that apelin limits the post-MI expression of ANP, suggesting a protective role of apelin in cardiac homeostasis (Figure 6C). Apelin also exerts its cardioprotective action by limiting cardiac fibroblast activation through sphingosine kinase 1 inhibition (24). Consistent with this report, we showed that apelin has a beneficial 

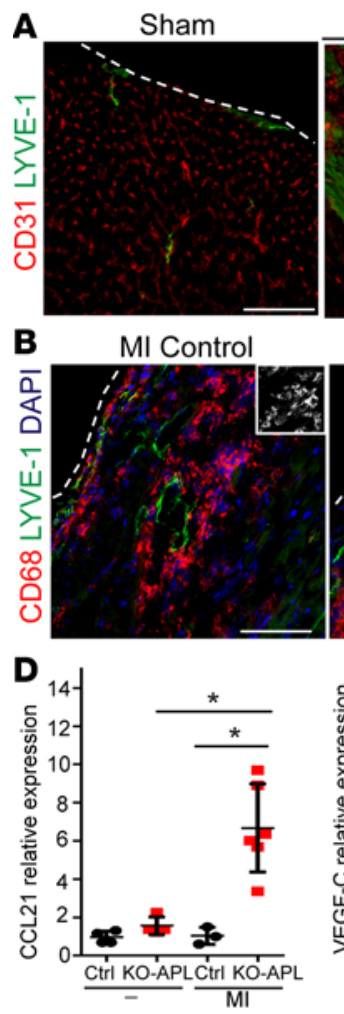

E
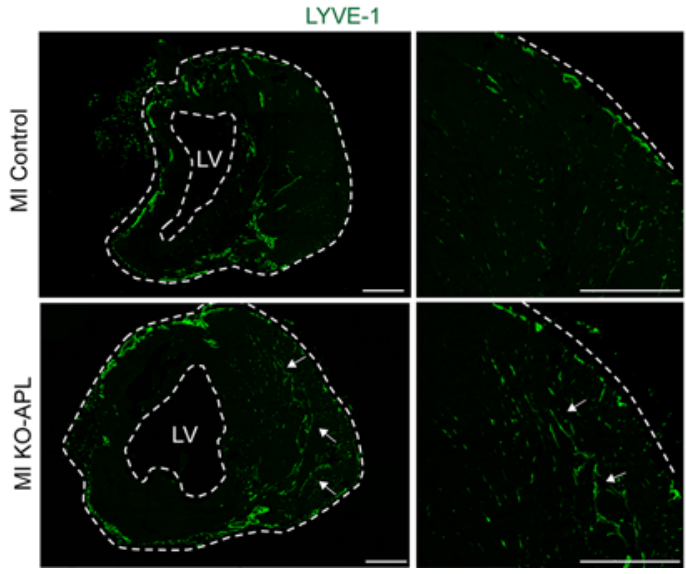

MI 4 days

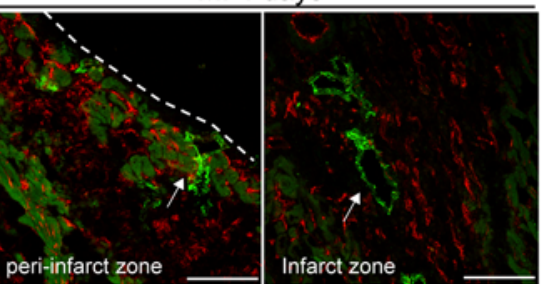

MI KO-APL
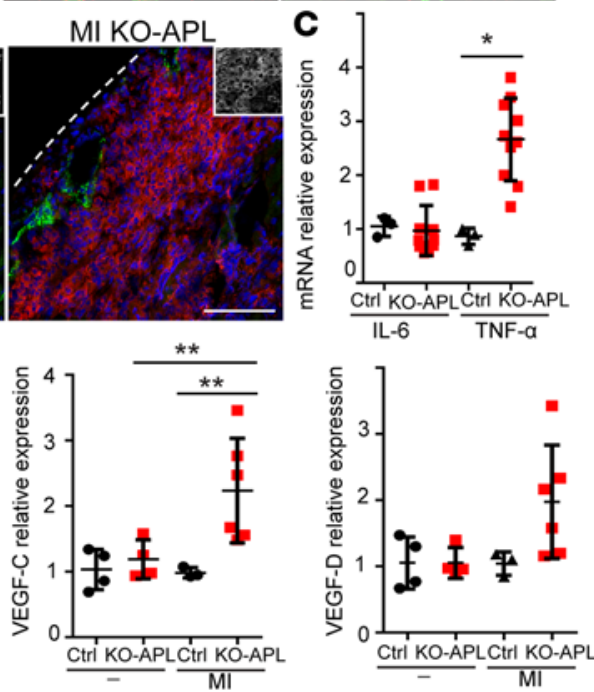

MI

Figure 5. Apelin-KO mice exhibit strong inflammatory and prolymphangiogenic responses after myocardial infarction. (A) Lymphatic remodeling in WT mice 4 days after myocardial infarction (MI) examined by LYVE-1 and CD31 immunostaining. Note the presence of highly dilated and hyperplastic lymphatic vessels in the peri-infarcted and infarcted zone. (B) Visualization of lymphatic vessels (LYVE-1+) and macrophages (CD68 $)$ in the infarct border zone 4 days after $\mathrm{MI}$ in apelin-KO versus control mice. Higher magnification is shown in the upper boxes. For scale, the dimensions of the higher-magnification boxes are $60 \times 60 \mu \mathrm{m}$. (C) Expression of inflammatory cytokines IL- 6 and $\operatorname{Tnf} \alpha 2$ days after MI ( $n=4$ per group; horizontal bars represent the mean $\pm \mathrm{SD}$ ). ${ }^{*} P<0.0002$. (D) Expression of the chemokine $C \mathrm{C} / 21$ and prolymphangiogenic factors Vegf- $C$ and Vegf- $D$ in apelin-KO mice 2 days after MI in apelin-KO mice versus control mice $(n=4$ per group; horizontal bars represent the mean $\pm \mathrm{SD}$ ). ${ }^{*} P<0.0003$, ${ }^{* *} P<0.002$. (E) Tile scan analysis by confocal microscopy of whole heart showing LYVE1-positive lymphatic vessels. Dotted lines delimit the heart and the left ventricle (LV). Lymphangiogenesis is further amplified in apelindeficient mice 6 weeks after Ml compared with control. Arrows show the localization of lymphatic vessels in the right ventricle. Statistical analysis was done with 1-way ANOVA with Bonferroni post-hoc test. Scale bars: $100 \mu \mathrm{m}$ (A and $\mathbf{B})$ and $500 \mu \mathrm{m}$ (E). APL, apelin.

effect on collagen, metalloproteases, hyaluronan synthase 1, and sphingosine kinase 1 expression (Figure 6D). Of note, the proinflammatory mediators $T N F \alpha$ and $I L 1 \beta$ were also reduced in the presence of apelin (Figure $6 \mathrm{E}$ ). These data correlate with a diminution in $\mathrm{CD}^{+} 8^{+}$macrophage infiltration in the ischemic heart in the presence of apelin (Figure 6, F and G). Importantly, lymphatic vessels acquired a regular morphology and wellshaped organization (Figure $6 \mathrm{H}$ ). Interestingly, the number and the dilation of lymphatic vessels were remarkably decreased, suggesting that apelin/APJ signaling promotes the maturation of developing lymphatic vasculature after cardiac ischemia (Figure 6, I-K). Taken together, our data highlight a beneficial role of apelin in the cardiac microenvironment associated with functional lymphatic vascular repair after MI.

Apelin regulates SIP secretion and cell-cell junction integrity in LECs. Apelin expression is strongly induced by hypoxic stress (25). We next deciphered the molecular mechanism by which apelin controls lymphatic vessel maturation by performing an in vitro study in LECs. First, we measured the effect of hypoxia on the expression of apelin in LECs. As expected, we found an upregulation of apelin (26) and the prolymphangiogenic receptor $C X C R 4$ (27) under hypoxic conditions versus normoxia (Supplemental Figure 7A). Apelin expression was induced after 8 hours under hypoxia in LECs (Supplemental Figure 7B), and the apelin peptide was detected at the LEC membranes at 24 hours by immunostaining (Supplemental Figure 7, C and D). As the integrity of the lymphatic endothelium is crucial to its function, we next investigated the consequence of hypoxic stress on the organization of lymphatic cell-cell junctions in vitro. Interestingly, hypoxia significantly decreased the abundance of VE-cadherin at cellular junctions, which is associated with cortical actin cytoskeleton disassembly (Figure 7, A and B). Therefore, we wondered whether apelin could modulate the barrier integrity of LECs in vitro under hypoxic conditions. To test this, LECs were transduced either with GFP- or apelin-lentiviral vector and then cultured under hypoxic conditions. Apelin expression was first confirmed in transduced LECs (Figure 7C). The presence of apelin prevented the loss of VE-cadherin expression in cell-cell junctions under hypoxic conditions (Figure 7, D and E). A similar finding was also observed in LECs in coculture with myoblast H9C2 cells transduced with apelin (Supplemental Figure 7E). Because VE-cadherin is the main regulator of vascular permeability, we validated that apelin prevents the permeability induced by hypoxia (Figure 7F). Altogether, our in vitro data demonstrate a beneficial role of apelin 
A

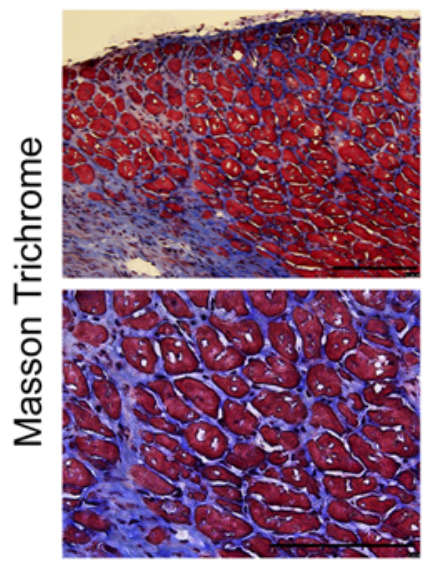

D
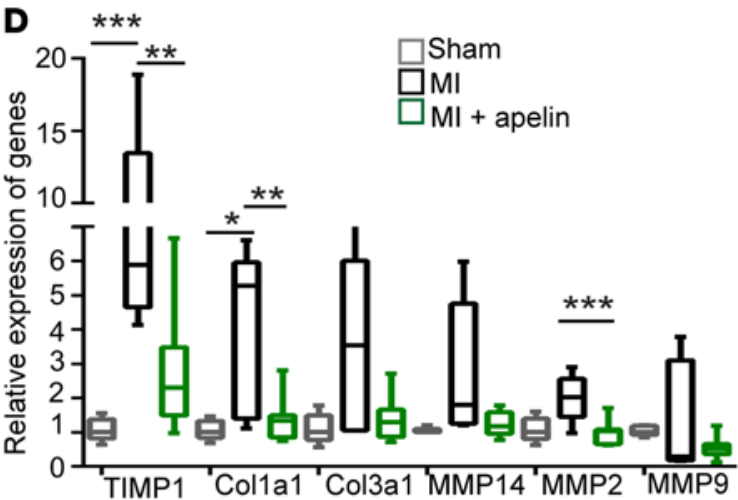

F

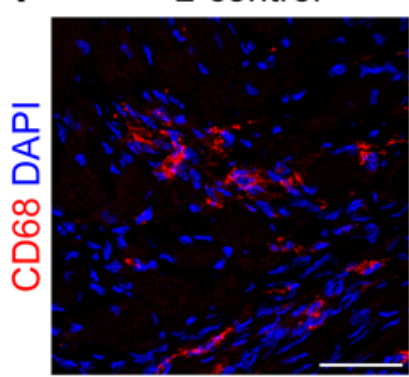

H

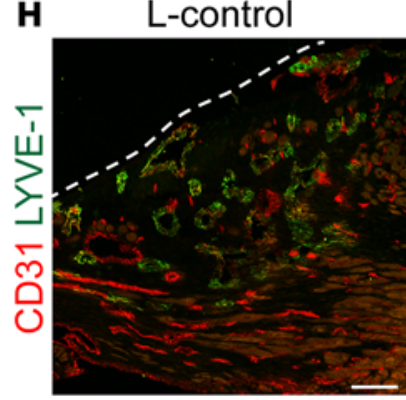

L-apelin

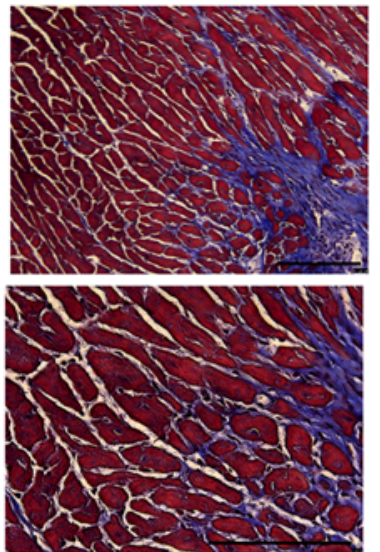

B
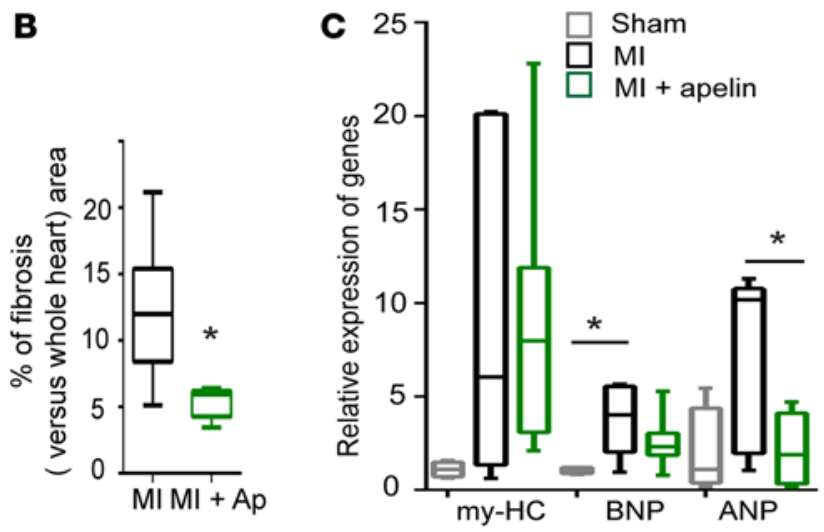

E
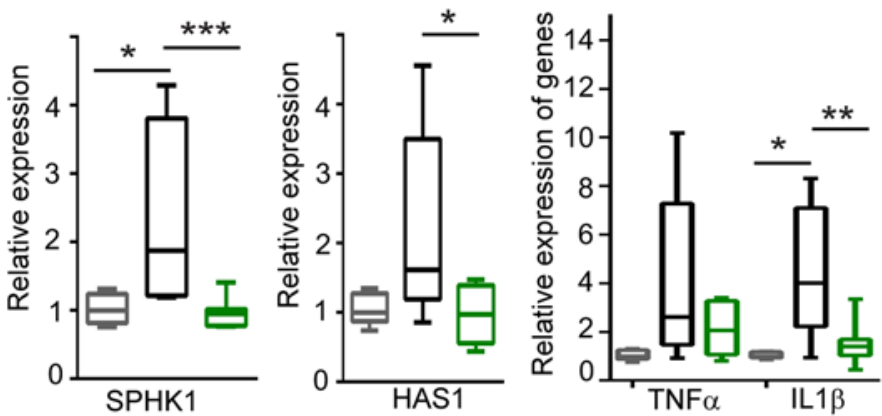

G

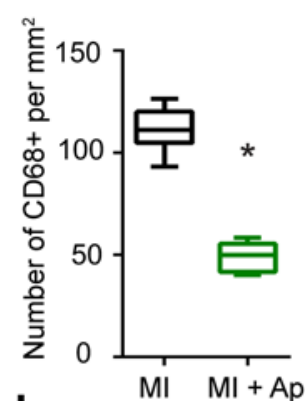

I

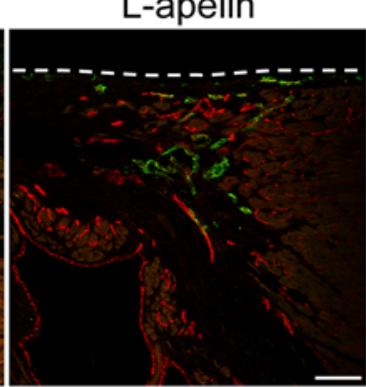

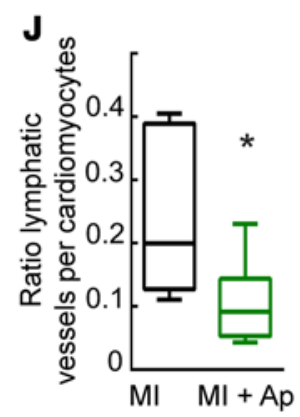

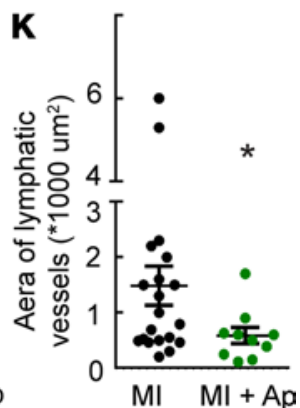

Figure 6. Apelin prevents fibrosis, inflammation, and pathological remodeling of lymphatic vasculature in chronic myocardial infarction. (A) Masson's trichrome staining in heart expressing control lentivector (L-control) or apelin lentivector (L-apelin). (B) Quantification of fibrosis ( $n=$ 5-10). ${ }^{*} P<0.01$. (C-E) Gene expression analysis related to cardiac hypertrophy (C), matrix remodeling (D), and inflammatory mediators (E) in heart of sham mice, or 6 weeks after myocardial infarction (MI) with or without treatment with the therapeutic $L$-apelin $(n=5-7)$. ${ }^{*} P<0.04$, ${ }^{* *} P<0.02$, ${ }^{* *} P<0.01$. (F) Visualization of CD68 ${ }^{+}$macrophages at the infarct border zone in heart transduced with L-apelin compared with control. (G) Quantification of $\mathrm{CD} 8^{+}$macrophages in the infarct border zone. ${ }^{*} P<0.05$. (H) Visualization of lymphatic vasculature 6 weeks after MI with or without treatment with the therapeutic L-apelin. Note the absence of hyperplastic and highly dilated lymphatic vessels observed in mice treated with L-apelin. (I) Lymphatic vessel density at the infarct border zone treated with L-apelin or L-control (values are the mean \pm SD). ${ }^{*} P<0.01$. (J) Ratio of the number of lymphatic vessels per cardiomyocytes $(n=6-10) .{ }^{*} P<0.05$. (K) Area of lymphatic vessels at the border of the infarct zone (values 
are the mean \pm SD). ${ }^{*} P<0.05$. Ap, apelin; my-HC, myosin heavy chain; BNP, brain natriuretic peptide; ANP, atrial natriuretic peptide; TIMP1, TIMP metallopeptidase inhibitor 1; Col1a1, collagen I; Col3a1, collagen III; MMP, metalloproteinase; SPHK1, sphingosine kinase 1; HAS1, hyaluronidase synthase 1. Statistical analysis was done with 1-way ANOVA with Bonferroni post-hoc multiple comparison test (C-E) or Student's $t$ test (B, G, I, J, and $\mathbf{K})$. Scale bars: $100 \mu \mathrm{m}$ (A and $\mathbf{H})$ and $50 \mu \mathrm{m}(\mathbf{F})$.

in the maintenance of barrier integrity of the lymphatic endothelium. Recent studies reported an important role of S1P in vascular and lymphatic junction maturation $(10,28)$. Therefore, we next wondered whether apelin in LECs could regulate the level of S1P to maintain the stability of lymphatic endothelium. S1P is generated intracellularly by the action of sphingosine kinase that phosphorylates sphingosine and is exported out of the cells by the S1P-specific transporter SPNS2 to exert an autocrine or paracrine action $(29,30)$. Interestingly, apelin induces the expression of the sphingosine kinase 2 and SPNS2 on LECs (Figure 7G). We validated our result in LECs constitutively expressing apelin and noted a 5-fold increase in gene expression (Figure 7H) and a significant elevation of S1P quantity in conditioned media (Figure 7I). Taken together, our results reveal a possible mechanism by which apelin modulates S1P secretion in LECs and maintains the barrier integrity of the lymphatic endothelium.

\section{Discussion}

Improving lymphatic network integrity has recently emerged as a crucial target for cardiovascular disorders. The principal challenge related to improvement of lymphatic function is to identify bioactive molecules possessing an inherent reparative ability. It has been clearly established that VEGF-C/VEGFR3 signaling contributes to cardiac lymphatic development $(3,31)$. Nevertheless, VEGF-C alone is not sufficient to restore the integrity of the post-MI lymphatic endothelium (2). Here, we found that apelin controls the functional stability of lymphatic endothelium in cardiac ischemia. In particular, our study highlights a potentially novel mechanistic insight into apelin-induced S1P secretion in LECs.

The heart contains a comprehensive lymphatic network mainly composed of blunted-end capillaries in the myocardium and larger collecting vessels located at the subepicardial surface of the heart. We demonstrated that those larger collecting vessels in adult heart are VEGFR3 ${ }^{\text {hi }} C D 31^{\text {lo }}$ podoplanin ${ }^{\text {hi }} \mathrm{NRP} 2^{\text {hi }}$, while intramyocardial lymphatic capillaries are negative for podoplanin. A lack of podoplanin in adult mice reduces dendritic cell migration to lymph nodes (32). The presence of podoplanin in cardiac collecting lymphatic vessels emphasizes the potential role of lymphatic vessels in facilitating the trafficking of immune cells in response to inflammation-related cardiovascular diseases. Intriguingly, the majority of lymphatic vessels in rat heart are intramyocardial lymphatic capillaries that drain fluid centrifugally towards the epicardium (2). We show in mouse heart a low level of intramyocardial lymphatic vessels compared with an abundant lymphatic network located at the subepicardial surface of the heart, suggesting anatomical differences in the lymphatic network between species. Interestingly, we and others $(33,34)$ observed an important network of lymphatic vessels in heart valves, suggesting that lymphatic vessels may contribute to resolving inflammation and fibrosis in valvular pathologies. Indeed, recent studies have reported a strong remodeling of lymphatic vasculature in various cardiovascular diseases (35), yet the molecular mechanisms underlying the maintenance of a functional lymphatic network in cardiac diseases are still poorly described. Our study reveals that the dilation of lymphatic vasculature occurs early after MI and correlates with edema and immune cell infiltration. Although the lymphatic vasculature is a key player in the immune response, its role in the acute phase of MI remains to be elucidated. Remodeling of the lymphatic system has been recently proposed to participate in edema and tissue fibrosis in $\mathrm{MI}(2,36)$. We provide evidence that the lymphangiogenic process occurs in the periphery of the ischemic zone 2 weeks after MI and extends to the right ventricle 6 weeks after MI. However, this newly formed lymphatic network associated with disrupted collagen fibers in the ischemic area is highly disorganized, tortuous, and dilated. This anarchic growth of lymphatic vessels correlates with the development of dysfunctional vessels (2). Therefore, we examined the mechanisms underlying a functional recovery of lymphatic vasculature. Apelin plays a critical role in protecting the cardiac microenvironment, regulating the behavior of extravascular cells (immune cells, fibroblasts) and blood vasculature. The observation that improvement of the post-MI cardiac lymphatic network requires apelin was first derived from the finding that the presence of the receptor APJ was only detected on activated lymphatic endothelium in ischemic heart, whereas no expression was found on quiescent vessels. Although apelin/APJ is not required during embryonic lymphatic development (15), our results highlight a restrictive role of apelin/APJ during pathological lymphangiogenesis after MI, suggesting that apelin/ 
A
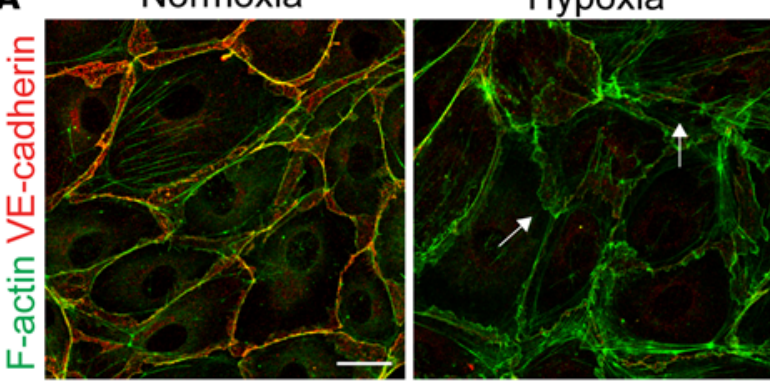

B

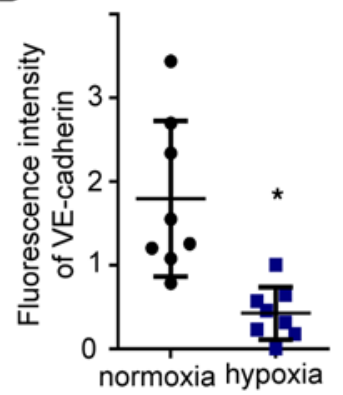

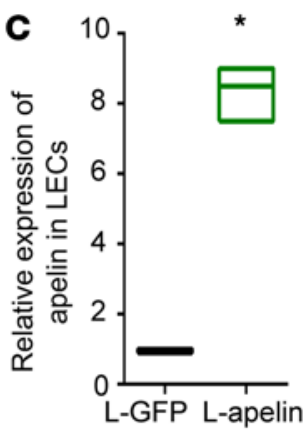

Hypoxia
E

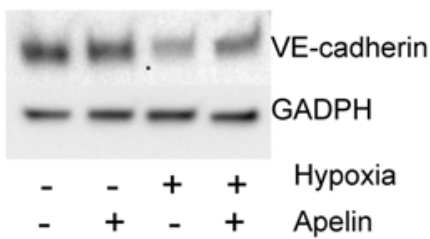

G

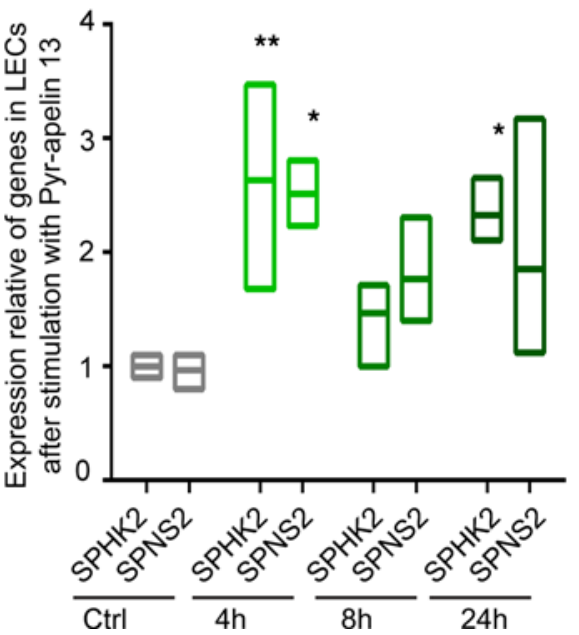

F

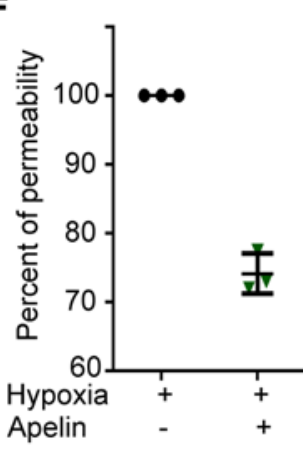

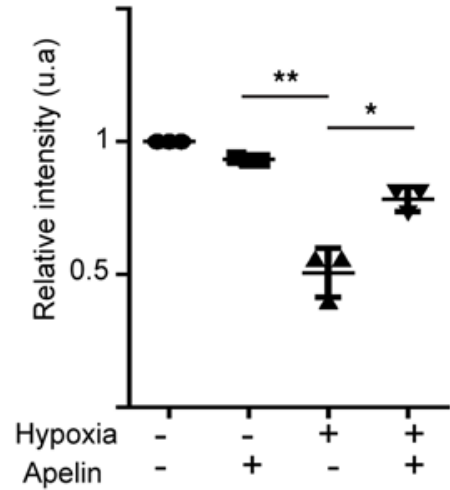
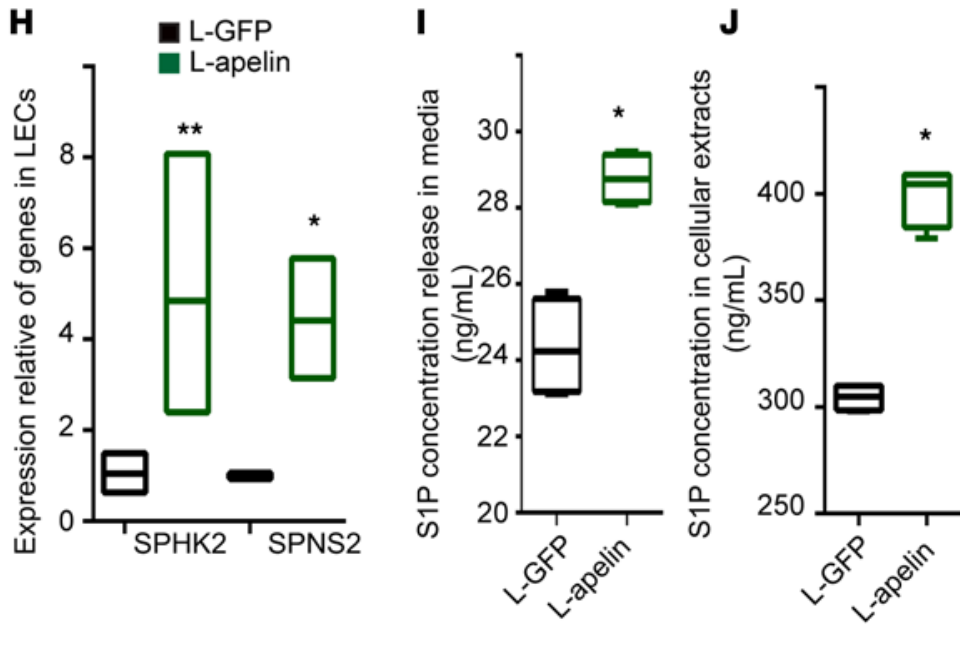

Figure 7. Apelin regulates S1P secretion and participates in the barrier integrity of the lymphatic endothelium in vitro. (A) Subconfluent lymphatic endothelial cells (LECs) were exposed to hypoxia or normoxia overnight. Note the reduction in VE-cadherin expression and loss of cortical actin cytoskeleton on newly formed cell-cell junctions between LECs under hypoxic conditions. (B) Quantification of VE-cadherin immunostaining from representative images shown in $\mathbf{A}$ ( $n=3$ independent experiments, data represent the mean \pm SD). ${ }^{*} P<0.05$. (C) Expression of apelin on LECs transduced by lentiviral vector GFP or apelin ( $n=3$ independent experiments). ${ }^{*} P<0.05$. (D) VE-cadherin immunostaining in LECs transduced by lentivector GFP or apelin cultured under hypoxic or normoxic conditions. Note the maintenance of VE-cadherin at cell-cell junctions in presence of apelin in hypoxic conditions. (E) Western blot analysis of VE-cadherin in different conditions ( $n=3$ independent experiments). ${ }^{*} P<0.02,{ }^{* *} P<0.009$. (F) Permeability assay with LECs under hypoxic conditions transduced with GFP or apelin. (C) Expression of sphingosine kinase 2 (SPHK2) and the transporter spinster homolog 2 (SPNS2) by 
RT-qPCR in LECs stimulated by the peptide apelin ( $n=3$ independent experiments, data represent the mean \pm SD). ${ }^{*} P<0.04$, ${ }^{* *} P<0.01$. (H) Expression SPHK2 and SPNS2 in LECs transduced with GFP or apelin lentivector ( $n=3$ independent experiments). ${ }^{* *} P<0.01$. (I and J) Sphingosine-1-P (S1P) secretion in media (I) and cellular extracts (J) of LECs ( $n=3$ independent experiments). ${ }^{*} P<0.05$. Statistical analysis was done with 1 -way ANOVA with Bonferroni post-hoc multiple comparison test (G and $\mathbf{H})$ or Student's $t$ test (B, C, E, F, I, and J). Scale bars: $20 \mu \mathrm{m}$ (A and $\mathbf{D})$.

APJ signaling may favor metabolic adaptation of LECs in the ischemic zone. Previous studies highlighted a strict spatiotemporal regulation of apelin/APJ during blood development. In the retina, apelin is strongly upregulated in the tip cells of developing blood vessels, while the expression of the receptor APJ is mainly observed in stalk cells during vessel remodeling $(37,38)$. Here, we frequently observed heterogeneous expression of APJ on activated LECs, indicating that APJ is tightly regulated during lymphatic growth. In light of the literature, we hypothesize that APJ is required on activated lymphatic endothelium, whereas its expression may be downregulated on stabilized lymphatic endothelium.

Remarkably, adult apelin-deficient mice exhibit baseline abnormalities of lymphatic vasculature associated with functional defects. Enlarged and hyperplastic lymphatic vessels in apelin-deficient mice were mainly observed at the subepicardial surface of the heart. This finding points out the clinical importance of this superficial lymphatic network in limiting cardiac interstitial edema, inflammatory cells, and tissue metabolites from the myocardium to maintain heart function. In addition, this alteration of lymphatic vasculature in apelin-KO mice was accompanied by a polarization of macrophages towards the M1 proinflammatory phenotype, suggesting a defect in the lymphatic barrier in protecting from edema and immune cell trafficking. These observations are in accordance with previous literature showing that apelin has an antiinflammatory action (39). Here, our data further indicate that the alteration of lymphatic vessels may account for the development of the proinflammatory condition. In line with these findings, we observe that the lack of apelin induces a deleterious microenvironment characterized by an increase in inflammatory mediators and immune cell infiltration after MI. Although apelin-deficient mice have normal cardiac function, these mice exhibit a susceptibility to heart failure and reduced heart contractility in aging mice $(40,41)$. Lymphatic vessel dysfunction associated with the accumulation of immune cells in cardiac tissue could in part explain the increase of susceptibility to MI in apelin-deficient mice. Despite the vascular (40) and lymphatic defects observed in apelin-deficient mice, we detected an increase in lymphatic vessels in these mice that overcomes the critical phase of acute MI. This observation is in line with the hypothesis that the activation of lymphatic vessels in early-phase MI may have a beneficial role in stimulating lymphangiogenic growth in the chronic phase of MI. Apelin has a pleiotropic action, facilitating cardiomyocyte contractility $(19,42)$ and angiogenesis $(21,43)$, but also limiting inflammation (39) and lowering blood pressure $(18,44)$. Although the expression of APJ argues for a direct role of apelin in lymphatic endothelium, all of these effects of apelin on the cardiac microenvironment may indirectly influence the lymphatic function. It would be of interest to investigate the function of APJ specifically in lymphatic endothelium by generating a lymph-specific APJKO mouse model. Taken together, using in vivo loss- or gain-of-function mouse models, our studies argue for a role of apelin in the stabilization of lymphatic vasculature in pathological settings.

Hypoxia induces strong expression of apelin in cardiomyocytes in vivo and in vitro (25). Although LECs are capable of producing apelin under hypoxic conditions in vitro, we cannot exclude the importance of cardiomyocytes as a potent source of apelin in the heart, arguing for a paracrine effect of apelin on lymphatic endothelium. Hypoxia is a driving force of tumor lymphangiogenesis (45). In that context, developing lymphatic vessels are more permeable and unstable, which is an aggravating factor in tumor metastasis. Hypoxia-induced angiogenesis and lymphangiogenesis is crucial to improve the healing of the infarcted zone. Of interest, hypoxia alters the stability of LEC junctions in vitro, indicating defective junctional and mechanical properties under hypoxic stress. Importantly, we observed a beneficial role of apelin in lymphatic barrier integrity. Furthermore, apelin limits VEGF-mediated hyperpermeability of BECs (12), indicating an important role of apelin in cell-cell junction stabilization.

The barrier integrity of lymphatic endothelium is crucial to limiting persistent edema and inflammation after MI. Among the few factors described to improve vascular function, the bioactive sphingolipid S1P is well known to control the integrity of the blood vasculature by enhancing adherens and tight junction formation (46-48). Recent work has shown that S1P also regulates the maturation of lymphatic cell-cell junctions in vivo (10). As lymphatic function is highly dependent on the junctional organization of lymphatic endothelium, we next wondered whether apelin could regulate S1P levels in LECs. LECs are the major source of S1P in lymph $(10,30)$. The concentration of S1P in lymph is dependent on the 
action of sphingosine kinase intracellularly and its export into the extracellular space. SPNS2 has recently been shown to specifically regulate S1P secretion into lymph by LECs (30). Here, we provide evidence that apelin regulates the expression of sphingosine kinase 2 and the transporter SPNS2 that results in the elevation of S1P. We have identified a potentially novel mechanism through which the biopeptide apelin regulates S1P secretion. S1P gradients have emerged as key regulators of the trafficking of many types of immune cells. Intriguingly, apelin-deficient mice display a proinflammatory status in heart tissue. Although we could not exclude a direct role of apelin in immune cells, an imbalance of the S1P gradient in vascular and/or lymphatic vasculature may explain this phenotype. It thus remains to be determined whether the level of S1P in lymph promotes lymphatic maturation and the trafficking of immune cells in mice following cardiac ischemia.

In conclusion, the present study highlights that apelin contributes to lymphatic vessel function, and thus cardiac homeostasis. It is particularly interesting to note that half of patients with heart failure have a preserved cardiovascular function but have multiple cardiac abnormalities such as ventricular and vascular stiffness. In that context, restoration of lymphatic function based on lentivector-associated lymphangiogenic therapy may be an interesting strategy to preserve a healthy cardiac microenvironment. In regard to these observations, we could propose a combination of VEGF-C and apelin as a lymphangiogenic therapy in ischemic disorders.

\section{Methods}

Details for materials and methods are available in the Supplemental Data.

Mouse strains. WT mice on the C57BL/6 background were provided by Janvier. Apelin-deficient mice have been previously described (41). Animal experiments were conducted in accordance with recommendations of the European Convention for the Protection of Vertebrate Animals used for experimentation. All animal experiments were performed according to the INSERM IACUC guidelines for laboratory animals' husbandry and have been approved by the local branch Inserm Rangueil-Purpan of the Midi-Pyrénées ethics committee. Apelin-KO mice were obtained from Philippe Valet. WT mice on the C57BL/ 6 background (provided by Janvier) were used as controls. All mice were from 8 to 10 weeks old. A complete description of the experimental procedure regarding $\mathrm{MI}$ is described in the Supplemental Data.

Flow cytometry and cardiac LEC isolation. Hearts cut into small pieces were dissociated with Liberase enzyme (Roche) for 10 minutes at $37^{\circ} \mathrm{C}$. Supernatants were filtered through a $100-\mu \mathrm{m}$ cell strainer into a 50-ml tube on ice. After lysis of erythrocytes (RBC Lysis Buffer, eBiosciences), cells were filtered through a 40- $\mu \mathrm{m}$ cell strainer. Cells were incubated with Fc blocking antibody CD16/CD32 (eBiosciences, catalog 16-0161) for 30 minutes, and then stained with the following antibodies on ice for 30 minutes: APC-CD31 (catalog 561814) and BB515-CD45 (catalog 564590) (both BD Pharmingen), LYVE-1-PE (R\&D Systems, catalog 223322), CD19-PercP-vio700 (catalog 130-102-237), CD3-PE-vio770 (catalog, 130-109-881), CD11b-PE-vio770 (catalog, 130-109-365) (all 3 from Miltenyi Biotec), and PercP/Cy5.5 CD206 (Biolegend, catalog 141715). Dead cells were excluded form analysis using Live/Dead Violet staining (Invitrogen). Flow cytometry was performed using a BD Fortessa cytometer and FACSDiva software analysis.

For cardiac LEC isolation, 9-10 hearts from WT mice were pooled to isolate cardiac LECs. We performed the same experiment for sham mice and post-MI mice. LECs from hearts were dissociated as described previously using LYVE-1-PE, CD45-BB515, and CD31-APC antibodies. LECs (LYVE$\left.1^{+} \mathrm{CD} 31^{+} \mathrm{CD} 45^{-}\right)$and BECs (LYVE-1 ${ }^{-} \mathrm{CD} 31^{+} \mathrm{CD} 45^{-}$) were separated using a BD influx cell sorter. Isolated cells from all experiments were used immediately for RNA preparation.

$R N A$ isolation and RT-qPCR analysis. Total RNA from in vivo-isolated cells and in vitro LECs were extracted with an RNAqueous-Micro Kit (Ambion). cDNA synthesis was carried out using superscript Vilo enzyme (Invitrogen). For primary LECs, we used High-Capacity cDNA reverse transcriptase (Applied Biosystems). RNA isolation from ischemic heart was carried out with TRIzol reagent (Ambion). RT-qPCR reactions were carried out on an ABI StepOne (Applied Biosystems).

Immunohistochemistry and image analysis. Cardiac lymphangiogenesis was determined from paraformaldehyde-fixed (PFA-fixed) paraffin or cryostat sections. Sections were numbered from the apex to the AV region in order to have a consistent and reproducible analysis: each slide was separated by $60 \mu \mathrm{m}$ with a gap of $250 \mu \mathrm{m}$ every 10 slides. Analysis on paraffin sections was done with anti-LYVE-1 antibody followed by secondary antibody (Impress reagent kit, Vector labs) and DAB (Vector labs). Immunofluorescence on 
cryosections was performed using the following antibodies: anti-mouse LYVE-1 (Rockland, 70R-LR005), neuropilin-2 (R\&D Systems, AF567), VEGFR3 (R\&D Systems, AF743), CD31 (BD, 553370), collagen I (Serotec, 2150-1410), and podoplanin (Developmental Studies Hybridoma Bank, 8.1.1).

Apelin and APJ antibodies have been previously described (49). Images were acquired using an LSM780 laser scanning confocal microscope (Zeiss) or Nanozoomer (Hamamatsu) for slide section imaging. Image analysis (vessel area, length, or number of branch points) was done with ImageJ software (NIH).

For whole-mount imaging, hearts were fixed with 4\% PFA for 6 hours and then washed with PBS for 2 days. Permeabilization was performed in $0.3 \%$ Triton-PBS solution for 2 days before adding anti-LYVE-1 antibody. After several washes, hearts were incubated with secondary antibody conjugated to Alexa-Fluor 488 (Jackson Immunoresearch) for 1 day. Post-staining fixation in 2\% PFA was performed. Whole-heart imaging was done using a Macrofluo microscope (Leica). Hearts were sectioned in 2 parts (ventral versus dorsal part) to image lymphatic vessels and collagen fibers under the multi-photon 7MP (Zeiss).

Cell culture. LECs (HDLECs, Promocell) were cultured in endothelial growth medium (EGM-MV2, Promocell) according to the manufacturer's instructions and maintained in culture for experiments up to passage 4. For hypoxia experiments, cells were maintained at $37^{\circ} \mathrm{C}$ in a humidified atmosphere under hypoxic $\left(1 \% \mathrm{O}_{2}\right)$ using a hypoxia chamber (Biospherix) or normoxic $\left(21 \% \mathrm{O}_{2}\right)$ conditions at indicated times in the figure legends. Cells were cultured at $80 \%$ confluence and reached full confluence under hypoxic conditions. Cells were fixed with 4\% PFA and antibody staining was performed for VE-cadherin (Santa Cruz, sc-6458). Actin cytoskeleton was visualized by phalloidin-488 (Cytoskeleton). Quantification of fluorescence intensity was performed with ImageJ software. All images were taken with the same gain and laser intensity. For the permeability assay, LECs were cultured under confluence for 2 days on cell culture inserts $(0.4 \mu \mathrm{m}$, Millicell) and then incubated for 24 hours under hypoxic or normoxic conditions as indicated in the figure legends. FITC-dextran, $70 \mathrm{kDa}(1 \mathrm{mg} / \mathrm{ml})$ was added and fluorescence intensity was measured 2 hours later in the lower chamber using a Tecan Infinite 500.

Statistics. Experiments are representative of at least 3 independent experiments, unless otherwise noted. Results are reported as mean \pm SD or mean \pm SEM as indicated in the figure legends. Graphs are presented either as dot plots, box-and-whisker plots, or boxes with minimum and maximum values. Statistical analyses were performed with Prism V6.0 (GraphPad Software) using 1-way ANOVA or unpaired 2-tailed Student's $t$ test, as described in the figure legends.

\section{Author contributions}

FT designed the research, performed experiments, analyzed the data, and wrote the manuscript. ERG performed experiments and analyzed the data. BM and PV provided the apelin-KO mice and antibodies against apelin and APJ. FH, ACG, FP, and FM participated in the research. DC performed surgery on mice. FV performed S1P dosage. ACP participated in the research and wrote the manuscript. BGS designed the research and wrote the manuscript.

\section{Acknowledgments}

Our thanks go to the Inserm US006 platform ANEXPLO Genotoul (Toulouse, France), Victorine Douin for help and advice on flow cytometry, Christiane Pecher and Alexia Zakaroff-Girard for the cytometry platform (TRI-genotoul, Toulouse), Madjid Zanoun and Danièle Daviaud from Imagery platform (TRIgenotoul, Toulouse), J.J. Maoret and F. Martins from GeT plateform Genotoul (Toulouse), and MetaToulLipidomique Core Facility (I2MC, Inserm 1048, Toulouse, France), MetaboHUB-ANR-11-INBS-0010.

This work was supported by Association pour la Recherche sur le Cancer (ARC), Région Midi-Pyrénées, AFM-Telethon. F.T. had a fellowship from ARC and from the Foundation Lefoulon-Delalande. E.R.G. had a fellowship from AFM-Telethon. F.H. had fellowships from the Région Midi-Pyrénées and from the Ligue Nationale Contre le Cancer, A.C.G from the Ligue Nationale Contre le Cancer.

Address correspondence to: Florence Tatin, I2MC INSERM UMR 1048, Toulouse Cedex, France. Phone: 33.561.323.818; Email: florence.tatin@inserm.fr.

FM's present address is: Laboratory of Tumor and Developmental Biology, GIGA-Cancer, Liège, Belgium. 
1. Alitalo K. The lymphatic vasculature in disease. Nat Med. 2011;17(11):1371-1380.

2. Henri $\mathrm{O}$, et al. Selective stimulation of cardiac lymphangiogenesis reduces myocardial edema and fibrosis leading to improved cardiac function following myocardial infarction. Circulation. 2016;133(15):1484-1497.

3. Klotz L, et al. Cardiac lymphatics are heterogeneous in origin and respond to injury. Nature. 2015;522(7554):62-67.

4. Losordo DW, et al. Gene therapy for myocardial angiogenesis: initial clinical results with direct myocardial injection of phVEGF165 as sole therapy for myocardial ischemia. Circulation. 1998;98(25):2800-2804.

5. Kastrup J, et al. Direct intramyocardial plasmid vascular endothelial growth factor-A165 gene therapy in patients with stable severe angina pectoris A randomized double-blind placebo-controlled study: the Euroinject One trial. J Am Coll Cardiol. 2005;45(7):982-988.

6. Tammela T, et al. Therapeutic differentiation and maturation of lymphatic vessels after lymph node dissection and transplantation. Nat Med. 2007;13(12):1458-1466.

7. D'Alessio S, et al. VEGF-C-dependent stimulation of lymphatic function ameliorates experimental inflammatory bowel disease. J Clin Invest. 2014;124(9):3863-3878

8. Cui Y, et al. Therapeutic lymphangiogenesis ameliorates established acute lung allograft rejection. J Clin Invest. 2015;125(11):4255-4268.

9. Yanagida K, Hla T. Vascular and immunobiology of the circulatory sphingosine 1-phosphate gradient. Annu Rev Physiol. 2017;79:67-91.

10. Pham TH, et al. Lymphatic endothelial cell sphingosine kinase activity is required for lymphocyte egress and lymphatic pattern ing. J Exp Med. 2010;207(1):17-27.

11. Yang P, Maguire JJ, Davenport AP. Apelin, Elabela/Toddler, and biased agonists as novel therapeutic agents in the cardiovascular system. Trends Pharmacol Sci. 2015;36(9):560-567.

12. Kidoya H, et al. Spatial and temporal role of the apelin/APJ system in the caliber size regulation of blood vessels during angiogenesis. EMBO J. 2008;27(3):522-534.

13. Kidoya H, Naito H, Takakura N. Apelin induces enlarged and nonleaky blood vessels for functional recovery from ischemia. Blood. 2010;115(15):3166-3174.

14. Kim JD, et al. Essential role of Apelin signaling during lymphatic development in zebrafish. Arterioscler Thromb Vasc Biol. 2014;34(2):338-345.

15. Kidoya H, et al. APJ regulates parallel alignment of arteries and veins in the skin. Dev Cell. 2015;33(3):247-259.

16. Berta J, et al. Apelin promotes lymphangiogenesis and lymph node metastasis. Oncotarget. 2014;5(12):4426-4437.

17. Sawane M, Kajiya K, Kidoya H, Takagi M, Muramatsu F, Takakura N. Apelin inhibits diet-induced obesity by enhancing lymphatic and blood vessel integrity. Diabetes. 2013;62(6):1970-1980.

18. Sato T, et al. Apelin is a positive regulator of ACE2 in failing hearts. J Clin Invest. 2013;123(12):5203-5211.

19. Scimia MC, et al. APJ acts as a dual receptor in cardiac hypertrophy. Nature. 2012;488(7411):394-398.

20. Masri B, van den Berghe L, Sorli C, Knibiehler B, Audigier Y. [Apelin signalisation and vascular physiopathology]. J Soc Biol. 2009;203(2):171-179.

21. Tempel D, et al. Apelin enhances cardiac neovascularization after myocardial infarction by recruiting aplnr ${ }^{+}$circulating cells Circ Res. 2012;111(5):585-598.

22. Gordon EJ, Rao S, Pollard JW, Nutt SL, Lang RA, Harvey NL. Macrophages define dermal lymphatic vessel calibre during development by regulating lymphatic endothelial cell proliferation. Development. 2010;137(22):3899-3910.

23. Sergeeva IA, Christoffels VM. Regulation of expression of atrial and brain natriuretic peptide, biomarkers for heart development and disease. Biochim Biophys Acta. 2013;1832(12):2403-2413.

24. Pchejetski D, et al. Apelin prevents cardiac fibroblast activation and collagen production through inhibition of sphingosine kinase 1. Eur Heart J. 2012;33(18):2360-2369.

25. Ronkainen VP, et al. Hypoxia inducible factor regulates the cardiac expression and secretion of apelin. FASEB J. 2007;21(8):1821-1830

26. Eyries M, et al. Hypoxia-induced apelin expression regulates endothelial cell proliferation and regenerative angiogenesis. Circ Res. 2008;103(4):432-440.

27. Schioppa T, et al. Regulation of the chemokine receptor CXCR4 by hypoxia. J Exp Med. 2003;198(9):1391-1402.

28. Camerer E, et al. Sphingosine-1-phosphate in the plasma compartment regulates basal and inflammation-induced vascular leak in mice. J Clin Invest. 2009;119(7):1871-1879.

29. Maceyka M, Spiegel S. Sphingolipid metabolites in inflammatory disease. Nature. 2014;510(7503):58-67.

30. Mendoza A, et al. The transporter Spns2 is required for secretion of lymph but not plasma sphingosine-1-phosphate. Cell Rep. 2012;2(5):1104-1110.

31. Mäkinen T, et al. Inhibition of lymphangiogenesis with resulting lymphedema in transgenic mice expressing soluble VEGF receptor-3. Nat Med. 2001;7(2):199-205.

32. Bianchi R, et al. Postnatal deletion of podoplanin in lymphatic endothelium results in blood filling of the lymphatic system and impairs dendritic cell migration to lymph nodes. Arterioscler Thromb Vasc Biol. 2017;37(1):108-117.

33. Kholová I, et al. Lymphatic vasculature is increased in heart valves, ischaemic and inflamed hearts and in cholesterol-rich and calcified atherosclerotic lesions. Eur J Clin Invest. 2011;41(5):487-497.

34. Niinimäki E, Mennander AA, Paavonen T, Kholová I. Lymphangiogenesis is increased in heart valve endocarditis. Int J Cardiol. 2016;219:317-321.

35. Vuorio T, Tirronen A, Ylä-Herttuala S. Cardiac lymphatics - a new avenue for therapeutics? Trends Endocrinol Metab. 2017;28(4):285-296.

36. Loukas M, Abel N, Tubbs RS, Grabska J, Birungi J, Anderson RH. The cardiac lymphatic system. Clin Anat. 2011;24(6):684-691.

37. Saint-Geniez M, Masri B, Malecaze F, Knibiehler B, Audigier Y. Expression of the murine msr/apj receptor and its ligand apelin is upregulated during formation of the retinal vessels. Mech Dev. 2002;110(1-2):183-186.

38. Papangeli I, et al. MicroRNA 139-5p coordinates APLNR-CXCR4 crosstalk during vascular maturation. Nat Commun. 2016;7:11268 
39. Sawane M, Kidoya H, Muramatsu F, Takakura N, Kajiya K. Apelin attenuates UVB-induced edema and inflammation by promoting vessel function. Am J Pathol. 2011;179(6):2691-2697.

40. Wang W, et al. Loss of apelin exacerbates myocardial infarction adverse remodeling and ischemia-reperfusion injury: therapeutic potential of synthetic apelin analogues. J Am Heart Assoc. 2013;2(4):e000249.

41. Kuba K, et al. Impaired heart contractility in apelin gene-deficient mice associated with aging and pressure overload. Circ Res. 2007;101(4):e32-e42.

42. Szokodi I, et al. Apelin, the novel endogenous ligand of the orphan receptor APJ, regulates cardiac contractility. Circ Res. 2002;91(5):434-440.

43. Cox CM, D'Agostino SL, Miller MK, Heimark RL, Krieg PA. Apelin, the ligand for the endothelial G-protein-coupled receptor, APJ, is a potent angiogenic factor required for normal vascular development of the frog embryo. Dev Biol. 2006;296(1):177-189.

44. Ishida J, et al. Regulatory roles for APJ, a seven-transmembrane receptor related to angiotensin-type 1 receptor in blood pressure in vivo. J Biol Chem. 2004;279(25):26274-26279.

45. Morfoisse F, et al. Hypoxia induces VEGF-C expression in metastatic tumor cells via a HIF-1 $\alpha$-independent translation-mediated mechanism. Cell Rep. 2014;6(1):155-167.

46. Lee MJ, et al. Vascular endothelial cell adherens junction assembly and morphogenesis induced by sphingosine-1-phosphate. Cell. 1999;99(3):301-312.

47. Lee JF, et al. Dual roles of tight junction-associated protein, zonula occludens-1, in sphingosine 1-phosphate-mediated endothelial chemotaxis and barrier integrity. J Biol Chem. 2006;281(39):29190-29200.

48. Liu Y, et al. Edg-1, the G protein-coupled receptor for sphingosine-1-phosphate, is essential for vascular maturation. J Clin Invest. 2000;106(8):951-961.

49. Picault FX, Chaves-Almagro C, Projetti F, Prats H, Masri B, Audigier Y. Tumour co-expression of apelin and its receptor is the basis of an autocrine loop involved in the growth of colon adenocarcinomas. Eur J Cancer. 2014;50(3):663-674. 